Gdańsk 2019, Nr. 40

https://doi.org/10.26881/sgg.2019.40.10

Svitlana Macenka

Lwiwer-Iwan-Franko-Universität / Uniwersytet Lwowski

https://orcid.org/0000-0003-1373-2887

\title{
Das Übermenschliche im Menschlichen: Der anthropologische Aspekt des Gesanges in den Erzählungen von E. T. A. Hoffmann
}

\begin{abstract}
Dank seiner Natürlichkeit, Einfachheit, Authentizität, Unmittelbarkeit, Reinheit, Sinnlichkeit und Naturverbundenheit spielt der Gesang in der Literatur der Romantik eine besondere Rolle. In den Erzählungen von E. T. A. Hoffmann wird er als ein musikalisches Geschehen betrachtet, das durch die Singstimme körperlich-sinnlich die Transzendenzerfahrung wiedergibt. Ihm wird deswegen die Funktion eines Mediums zugesprochen, welches die Resonanz des Übermenschlichen (des Unaussprechlichen - Göttlichen oder Dämonischen) im Inneren eines Menschen schafft.

Schlüsselwörter: romantisches Menschenbild, romantischer Gesang, Gesangsstimme, E. T. A. Hoffmann

Superhuman in Human: Anthropological Aspect of Singing in E. T. A. Hoffmann's Short Stories. Due to its naturalness, simplicity, authenticity, ingenuousness, pureness, sensuality and connection to nature, singing plays a special role in the literature of Romanticism. In E. T. A. Hoffmann's short stories, singing is seen as a musical event, where singing voice helps convey transcendent experience through physical and sensory sensations. This is why singing is endowed with the function of a medium which creates the resonance of superhuman (indescribable - divine or demonic) in the inner world of a person.
\end{abstract}

Keywords: romantic conception of human, romantic singing, singing voice, E. T. A. Hoffmann

Der romantische Gesang, dessen besonderen ästhetischen Status Roland Barthes hervorgehoben hat (Der romantische Gesang, 1976), ist, wie man insbesondere den Werken von E. T. A Hoffmann entnimmt, auch als ein aufschlussreiches literarisches Phänomen zu betrachten. Durch die Gefühlsintensität, Konzentration auf das subjektive Erlebnis, den Schwebezustand, der das Wunderbare erahnen lässt, veranschaulicht das Gesangsgeschehen die Leitgedanken der romantischen Ästhetik. Auch der romantische Erkenntnisweg der Einfühlung wird durch den Gesang verdeutlicht, wobei das Innere des Menschen zum Schauplatz der Ereignisse und zum Erfahrungsstifter wird. Als eine Ausdrucksweise von innigsten Gefühlen und gesteigerten Emotionen gibt der Gesang die wichtigsten Empfindungen des menschlichen Gemüts wieder, verkörpert das Ideal der romantischen Vorstellungswelt und fungiert 
im literarischen Text deshalb als Mittelpunkt der Handlung. Auch poetologisch spielt der Gesang für die romantische Literatur eine besondere Rolle: Verstärkt durch eine auch im imaginären Gesang spürbare Melodie erreicht der Text das Unsagbare und potenziert infolgedessen den sprachlichen Ausdruck. Dadurch entsteht die Möglichkeit, zu einer Art reiner Seligkeit zu gelangen. Es ist bemerkbar, dass im literarischen Gesangsgeschehen der Mensch von einer anderen, höheren Stimme durchtönt wird. Deswegen erfährt er die Wirklichkeit anders als im Horizont des Ich-zentrierten Bewusstseins. Damit kann man den Gesang als ein wichtiges Medium der Selbsterfahrung auffassen.

Der Gesang wurde auch im damaligen Diskurs der Anthropologie zum Gegenstand der Überlegungen (J. G. Herders Ideen zur Philosophie der Geschichte der Menschheit, 1791; I. Kants Kritik der praktischen Vernunft, 1788 und seine Menschenkunde oder philosophische Anthropologie, 1831; Versuch einer Darstellung des animalischen Magnetismus als Heilmittel von C. A. F. Kluge, 1815). Daher wird dieser anthropologische Diskurs für die Erörterung des literarischen Gesangsgeschehens herangezogen, um die Frage der naturhaft-sinnlichen Ausdrucksformen zu erörtern und auf diesem Weg das romantische Menschenbild zu verdeutlichen. Es handelt sich dabei insbesondere um das Verhältnis zwischen Seele und Geist, die Rolle der Sinnlichkeit als Verbindung des Menschen mit der Außenwelt und die harmonische Belebung des menschlichen Körpers.

E. T. A. Hoffmann, der die Instrumentalmusik als das adäquate Medium des autonomen Klangs bevorzugte, hat dabei den Gesang dem älteren Modell des Gefühlsausdruck zugeordnet.

Sollte, wenn von der Musik als einer selbständigen Kunst die Rede ist, nicht immer nur die Instrumental-Musik gemeint sein, welche, jede Hilfe, jede Beimischung einer andern Kunst (der Poesie) verschmähend, das eigentümliche, nur in ihr zu erkennende Wesen dieser Kunst rein ausspricht? Sie ist die romantischste aller Künste, beinahe möchte man sagen, allein echt romantisch, denn nur das Unendliche ist ihr Vorwurf. (Hoffmann 1908: 55)

Der Schriftsteller trennt, wie Andreas Käuser formuliert, die nichtsprachliche Musik von der sprachlichen Musik des Gesanges, ,,in dem eben das ältere Zeichenmodell überlebt“ (Käuser 1999: 192). Deswegen behauptet er:

In dem Gesange, wo die Poesie bestimmte Affekte durch Worte andeutet, wirkt die magische Kraft der Musik wie das wunderbare Elixier der Weisen, von dem etliche Tropfen jeden Trank köstlicher und herrlicher machen. (Hoffmann 1908: 55)

Für E. T. A. Hoffmann war die metaphysische Einbindung der Musik von besonderer Bedeutung, war er doch überzeugt, dass die Musik den Menschen über den Alltag erhebt und unendliche Sehnsucht nach einem transzendenten Zustand erzeugt. Dadurch lässt sich erklären, dass für ihn neben der Instrumentalmusik auch der Gesang als romantisch gelten konnte. Die Musik wird in der Romantik zur bedeutenden Sprache des Inneren, zum Lied ohne Worte, zur absoluten Musik, welche Instrumentalmusik dem Gesang „gleich“ macht.

Derart betrachtet vertieft das musikalische Gesangsgeschehen die dualistische Spannung des Hoffmann'schen Menschenbildes. Dadurch wird nicht nur die menschliche Duplizität (diesen Begriff verwendet E. T. A. Hoffmann in den Seltsamen Leiden eines Theater-Direktors in der Bedeutung: Doppelheit, Zweiheit der Welt und des Menschen) inszeniert, sondern 
gleichzeitig auch der kämpferische Selbstfindungsprozess eingeleitet. Es gilt, dass der Gesang das Innerliche ausdrückt und dass der Mensch in ihm seinem eigenen Sein begegnet. Dementsprechend wird auch dem Gesang eine transzendierende Funktion zugesprochen. In Beethovens Instrumental-Musik formuliert der Schriftsteller das in der Weise, dass im Gesang durch Worte bestimmte Affekte angedeutet werden.

Jede Leidenschaft - Liebe - Hass - Zorn - Verzweiflung etc., wie die Oper sie uns gibt, kleidet die Musik in den Purpurschimmer der Romantik, und selbst das im Leben Empfundene führt uns hinaus aus dem Leben in das Reich des Unendlichen. (Hoffmann 1908: 55-56)

Diese Erhebung des Menschen durch den Gesang ins „Reich des Unendlichen“ spiegelt sich als Resonanz des Übermenschlichen (Unaussprechlichen, Göttlichen oder Dämonischen) im Inneren des Menschen wider und eröffnet in ihm verborgene innere Räume. Somit erfüllt der Gesang auch eine vermittelnde Funktion zwischen dem Übermenschlichen und Menschlichen, Geist und Seele, innerer und äußerer Welt, Mensch und Natur. Diesen vom Gesang losgelösten inneren Vorgang kann man mit der Kant'schen Formulierung als ein Spüren der „Erhabenheit unserer eigenen übersinnlichen Existenz" bezeichnen (Kant 1915: 114). Der Gesang in seinem ästhetischen Wohlgefallen lässt den Menschen einen bestimmten Aspekt dessen, was er ist, im Vollzug der entsprechenden „Spontaneität“ spüren, der sonst verdeckt bleibt. Dieses Gefühl kommt als ein Moment des Begreifens zur Geltung.

In der Erzählung Der Kampf der Sänger aus der Erzählsammlung Die Serapions-Brüder, in der die Grenzen zwischen Gegenwart, Geschichte und Fiktion verschwimmen, wird am Anfang eine harmonische innere Vision geschildert, durch die „die anmutigsten Bilder eines höheren Lebens voll Glanz und Herrlichkeit“ und der „liebliche Gesang“" zum Vorschein kommen (Hoffmann 1912a: 22-23). Mit dem gemeinsamen herrlichen Gesang aller am Wettbewerb teilnehmenden Meistersänger disharmoniert das gewaltige Lied des „wilden“ Sängers Heinrich von Ofterdingen. Und nur das Zusammensingen aller Meister bändigt die Gewalt seines Erklingens und stellt die Ordnung wieder her.

Der Gesang der Meister versauste im Widerhall, schwarze Nebel legten sich über Wald und Wiesenplan, und hüllten alles ein in finstre Nacht. Da stieg ein in milchweißem Licht herrlich funkelnder Stern empor aus der Tiefe und wandelte daher auf der Himmelsbahn, und ihm nach zogen die Meister auf glänzenden Wolken singend und ihr Saitenspiel rührend. Ein flimmerndes Leuchten zitterte durch die Flur, die Stimmen des Waldes erwachten aus dumpfer Betäubung und erhoben sich und tönten lieblich hinein in die Gesänge der Meister. (Hoffmann 1912a: 26-27)

Die Existenz der Sänger wird somit als höchst harmonisch im Einklang mit der Natur dargestellt, mit frommer Liebe und Eintracht erfüllt, jeder von ihnen vervollkommnet den Gesang der anderen und betrachtet die eigene Weise als „lieblich“ nur im allgemeinen Zusammenklang. Jedoch werden durch den individuellen Gesang auch die unterschiedlichen Charaktere der Meistersänger erkennbar:

Bewies sich Heinrich Schreiber gelehrt und tiefsinnig, so war Johannes Bitterolff voller Glanz und reich an kunstvollen Gleichnissen und Wendungen. Heinrich von Ofterdingens Lieder gingen durch die innerste Seele, er wußte, selbst ganz aufgelöst in schmerzlichem Sehnen, in jeder Brust die tiefste 
Wehmut zu entzünden. Aber oft schnitten grelle häßliche Töne dazwischen, die mochten wohl aus dem wunden zerrissenen Gemüt kommen, in dem sich böser Hohn angesiedelt, bohrend und zehrend wie ein giftiges Insekt. (Hoffmann 1912a: 28)

Der erfahrenste von allen, Wolfframb von Eschinbach, drückte sich im Singen klar und weise aus. Er verstand, wenn er sang, jeden mitzureißen, so dass jeder „mit ihm wie auf den glänzenden Wogen eines schönen Stroms, bald sanft dahergleitend, bald kämpfend mit den sturmbewegten Wellen, bald die Gefahr überwunden, fröhlich hinsteuernd nach dem sichern Port [wallte]“"(Hoffmann 1912a: 29). Dadurch, dass Wolfframb von Eschinbach bei vielen Meistern gelernt hatte, sich den Zugang zu den Liedern durch Lektüre verschaffte und einen tiefen Glauben besaß, wurde er weise und einsichtsvoll. (Es heißt bei E. T. A. Hoffman (1912a: 29): „Licht strömte in sein inneres Gemüt hinein“). Das Licht unterstreicht die hohe geistige Stufe seiner persönlichen Entwicklung, sein Erkennen der eigenen künstlerischen Mission, im Gesang das Menschendasein nach dem Klang der göttlichen Natur auszurichten. Deswegen spürte er im Gesang von Heinrich als erster dessen Problem, das Hoffmann als „das unruhige zerrissene Wesen“ bezeichnet. Ofterdingens Lieder klagten über „die unermeßliche Qual des irdischen Lebens und glichen oft dem jammernden Wehlaut des auf den Tod Wunden, der vergebens hofft auf Erlösung im Tode" (ebd.: 30). Er schwankte zwischen Innigkeit und Hohn, Qual und Freude am Schmerz, Lebensverachtung und der Ambition, alle anderen zu übertreffen.

Im Unterschied zu Wolfframb von Eschinbach geht es bei Heinrich von Ofterdingen um sein subjektives Inneres, sein Gemüt, das nach dem Geistigen und seiner leitenden Kraft zwar strebt, dabei jedoch hofft, das Wahre nur durch Ahnung und allein durch gute Anlagen dereinst irgendwann zu erreichen. Wie die meisten Künstlergestalten bei E. T. A. Hoffmann ist er psychisch gefährdet. Die ihm selbst eigene Ambivalenz verkörpert der vor ihm plötzlich erschienene Fremde, der bei Heinrich einerseits Grauen und Abscheu erweckt, andererseits ihn anzieht. Die „absonderlichen“ Reden des Fremden hören sich wie „seltsame Lieder" an. Um mit den Worten Roland Barthes' zu sprechen: Es sang in ihm „einfach, ungeheuerlich, an der Grenze des Möglichen“ (Barthes 1990: 287). Der französische Philosoph spricht im Zusammenhang mit dem romantischen Gesang über eine mögliche Unterteilung der Stimmen.

Diese Unterteilung, von der er mitunter heimgesucht wird, ist freilich keine nach Geschlecht oder nach sozialen Rollen. Es ist eine andere Unterteilung: Sie stellt der schwarzen Stimme der Übernatur oder der dämonischen Natur die reine Stimme der Seele entgegen, und zwar nicht als religiöse, sondern als menschliche, allzu menschliche. (Barthes 1990: 287)

Dabei ist die Stimme des Bösen „ohne Ort“, „eine nirgends entspringende Stimme“ (Barthes 1990: 287), die auch die Stimme des Sängers dementsprechend zu einer ortlosen Stimme zu machen versucht. Für den Körper als vertrauten Stimmenort bedeutet das eine Art Verwirrung und Krankheit. Späterhin findet die negative Persönlichkeitsveränderung von Heinrich statt. Er bezeugt nun unverhohlen „Hohn“, „Unmut“ und Langeweile gegenüber seinen Mitsängern. Sein eindrucksvolles Lied, mit dem er bei einem Wettsingen siegte, singt er bezeichnenderweise in der Mitte des Kreises: 
Es war als schlüge er mit seinen gewaltigen Tönen an die dunklen Pforten eines fremden verhängnisvollen Reichs und beschwöre die Geheimnisse der unbekannten dort hausenden Macht empor. Dann rief er die Gestirne an, und indem seine Lautentöne leiser lispelten, glaubte man der Sphären klingenden Reigen zu vernehmen. (Hoffmann 1912a: 43)

Der Kreis und der „klingende Reigen“ weisen auf die Geschlossenheit des Heinrich'schen Liedsinns hin und bringen implizit seine Gestalt mit der Gestalt Kreislers in Verbindung wie Roland Barthes erklärt:

Jeder Augenblick dieser Reise ist gleichsam auf sich selbst zurückgewendet, blind, jedem allgemeinen Sinn, jeder Schicksalsvorstellung, jeder geistigen Transzendenz verschlossen: im Grunde ein reines Umherirren, ein Werden ohne Zielrichtung: das Ganze, insofern es auf einen Schlag und endlos wieder von vorne beginnen kann. (Barthes 1990: 291)

Als Eschinbach seine „wunderbare Weise“ und emporsteigenden Gedanken durchaus würdigt, äußert er auch die Befürchtung: ,solch ein Gesang könne nicht herausströmen aus dem rein menschlichen Gemüt, sondern müsse das Erzeugnis fremder Kräfte sein" (Hoffmann 1912a: 45). Er wirft seinem Mitstreiter die Entsagung aller Liebe vor und bezeichnet ihn als „verirrten Wanderer in der Wüste" (ebd.: 45). Allmählich verwandelt sich Heinrich von Ofterdingen zu einem Medium, durch das die „unbekannten Mächte“ singen, obwohl er selbst der Sänger bleibt. Somit gibt er auch einen wesentlichen Aspekt des serapiontischen Prinzips auf, das wahrhafte innere Schauen, das Wolfframb verkörpert, der sich bemüht, „nur das zu singen, was meine Brust mit freudiger, süßer Wehmut ganz und gar erfüllt" (ebd.: 55). Dagegen lernt Heinrich, auf die tief empfundene eigene Begeisterung zu verzichten. Er kommt durch seinen inneren Wandlungsprozess nicht aus seiner psychischen Gefährdung heraus, obwohl ihm der in frommen Liedern errungene Sieg von Eschinbach über die dunklen Mächte hilft. Das Überirdische in dieser Erzählung wird von Transzendenz dadurch konterkariert, dass der zerrissenen Gestalt Ofterdingens die Harmonie und persönliche Integrität Wolfframbs von Eschinbach gegenübergestellt wird (vgl. Schmidt 2009: 34).

Die Erzählung behandelt also die Problematik der vertikalen Einordnung: das Emporheben des Menschen durch den Gesang. Die Ausgangssituation zeigt Heinrich von Ofterdingen schon auf der Höhe seiner Kunstmeisterschaft, womit er sich jedoch nicht zufrieden gibt und daher weiter versucht, sich dem Absoluten anzunähern. Der Arzt Carl Alexander Ferdinand Kluge erklärte diesen Zustand in seiner Schrift Versuch einer Darstellung des animalischen Magnetismus, 1811, folgendermaßen:

Weicht die ganze Sinnlichkeit zurück, so tritt der Mensch aus der Verbindung mit der Aussenwelt und geht zur inneren Dunkelheit über [...] Herausgetreten aus dem Leben in den Aussendingen und in sich selbst versunken, steht der Mensch hier an der Grenze zweier sehr verschiedenen Welten, an der dunklen Pforte zum Übergang in ein höheres, besseres Seyn. (Kluge 1811: 109)

Die Grenzhaftigkeit seiner Person macht Heinrich von Ofterdingen zu einer verirrten, zwischen Diesseits und Jenseits schwebenden Seele. Wie Paola Mayer formuliert:

Wer sich über die alltägliche, menschliche Sphäre hinauswagt, verirrt sich in einer Welt ohne Wegweiser, in der das Göttliche und das Dämonische einander täuschend ähneln. Während aber das erste sich dem Menschen entzieht, kommt das letzte auf ihn verlockend zu mit dem Ergebnis, dass der Künstler nur 
selten und nur durch wahrhaft übermenschliche Wachsamkeit und Stärke einer Verstrickung mit dem Dämonischen entgehen kann. Das von Wolfframb erwähnte „unheimliche Grauen“ ist Reaktion auf das überwältigende Zweideutige, Inkommensurable der übersinnlichen Sphäre; es ist ein unbewußtes Wissen, dass man dort nicht mehr zu Hause ist, ein heilsames Zurückschrecken, da es den Menschen vor einer solchen Verirrung bewahren soll. (Mayer 2000: 64)

Der Gesang dient dabei einerseits als ein Mittel der Wirkung des Übermenschlichen im Menschlichen. Er verkündigt das Geheimnis der menschlichen Natur, gibt es aber nicht preis. Andererseits trägt der Gesang auch zur Harmonisierung des menschlichen Inneren bei; dabei betont er wesentlich den schwebenden Zustand des Menschen zwischen Seele und Geist.

Auch die sozialen Verhältnisse auf der Wartburg werden durch den Gesang veranschaulicht, und zwar überwiegend als ein harmonisches Zusammenklingen ,im lieblichsten Wohllaut" (Hoffmann 1912a: 28), eine im schöpferischen Sinne produktive Kommunikation. So passt Wolfframb von Eschinbach, der von inneren Konflikten frei ist, ohne Reibung in das gesellschaftliche Normsystem. Als anerkannter Meistersänger ist Heinrich von Ofterdingen in die Gemeinschaft der Dichter ebenfalls gut integriert. Die unauflösliche Ambivalenz aber, die er als Künstler in sich verspürt, seine höheren Ideale und niedere sinnliche Liebe, gefährdet sein soziales Verhalten, was als „grelle häßliche Töne“ (ebd.), „gewaltige Töne" (ebd.: 43) zur Geltung kommt. Einerseits bleiben der Künstler und sein Werk durch die objektive äußere Welt bestimmt, andererseits führt die poetische Vorstellungskraft durch die intensivste innere Tätigkeit zu einer Annäherung an das Absolute, das aber nicht erreicht werden kann, da der Mensch an die Realität gebunden ist. Das Motiv des Gesangswettbewerbs, das vor allem die Idee des Beherrschens künstlerischer Techniken verkörpert, bedeutet auch Ruhe und Überlegenheit im Leben. Das Streben nach höchster Form und Technik des Gesanges ist untrennbar mit den Wünschen nach Macht und leidenschaftlicher Liebe verbunden.

Darüber hinaus werden mit dem Gesang auch Reflexionen über die Geschlechtsdifferenzierung in die Erzählungen von E. T. A. Hoffmann eingebracht. Da in einigen Texten (zum Beispiel, Don Juan und Der Rat Krespel) ,insbesondere weibliche Künstlerfiguren über eine den männlichen Musikern/Komponisten dieser Texte ebenbürtige Genialität" verfügen, wird beim Schriftsteller das Hinausgehen ,über die ansonsten in der romantischen Literatur vorherrschende Asymmetrie der Geschlechterverhältnisse" festgestellt (Lubkoll/Neumeyer 2015: 268). Trotzdem enden die Verhältnisse zwischen Mann und Frau, die seelisch in der Kunst verwandt sind, mit dem Tod der Künstlerinnen, da sie zum Objekt sexuellen Begehrens des Mannes werden. In diesem Zusammenhang weist Christine Lubkoll auf das Werk Ideen zu einer Ästhetik der Tonkunst von Christian Friedrich Daniel Schubart aus dem Jahr 1806 hin, in dem das Instrumentalspiel als männlich konnotiert und der Gesang als natürliche Domäne weiblicher Musikalität betrachtet wird (Lubkoll 1995: 66). Laut Schubart ist die Menschenstimme

ganz natürlich Urton, und alle übrigen Stimmen der Welt sind nur ferner Nachhall dieser göttlichen Urstimme. Die Menschenkehle ist das erste, reinste, vortrefflichste Instrument in der Schöpfung. Ein natürlich schönsingendes Bauernmädchen rührt mehr, als der erste Violinist der Welt. (Schubart 1969: 335) 
Die Sängerinnen verfügen über mächtige und reine Töne, mit denen sie die Hörer verzaubern, die männlichen Musiker unterliegen häufig einer Verstimmung. In der Forschungsliteratur wird das dadurch erklärt, dass die Verbindung von weiblichem Körper und Singstimme durch den spätaufklärerischen Natur-Kultur-Diskurs bedingt ist, der das physiologische Anderssein und die Natürlichkeit der Frau postuliert, während die Verstimmtheit der Komponisten aus dem physikalischen Diskurs der Akustik rührt (Lubkoll/Neumeyer 2015: 402).

In der Erzählung Die Automate hört Ferdinand „die herrliche göttliche Stimme eines Weibes“, die in ihm ein „nie geahntes Gefühl“ hervorruft (Hoffmann 1912b: 96). Den wunderbaren, klaren (die Töne erklangen „wie helle Kristallglocken“), teils klagenden, teils sehnenden Gesang empfindet Ferdinand fast körperlich, physisch, da „ein unnennbares Entzücken“ sein Inneres durchbebte, der Schmerz krampfhaft seine Brust zusammenzog, sein Selbst ,in namenloser, himmlischer Wollust [unterging]“. Seine Seele verwandelte sich ganz in ein Ohr (ebd.). Das Ganze wiederum wird als ein „holdseliges Traumbild“ dargestellt. Dieser Zustand wird als Eröffnung seiner „inneren Stimme“ verdeutlicht:

Es ist die psychische Macht, die die Saiten in unserm Innern, welche sonst nur durcheinander rauschten, anschlägt, daß sie vibrieren und ertönen, und wir den reinen Akkord deutlich vernehmen; so sind wir aber es selbst, die wir uns die Antworten erteilen, indem wir die innere Stimme durch ein fremdes geistiges Prinzip geweckt, außer uns verständlicher vernehmen und verworrene Ahndungen, in Form und Weise des Gedankens festgebannt, nun zu deutlichen Sprüchen werden. (Hoffmann 1912b: 105-106)

Das bedeutet, dass der Gesang eine psychische Wirkung auf den Hörenden ausübt, dass er als ein musikalisches Geschehen eine besondere kommunikative Situation zwischen den realen und idealen Persönlichkeitsbildern wiedergibt. Es handelt sich also um einen psychischen Prozess in Form von Resonanz („psychische Macht“), evoziert durch ein fremdes „geistiges Prinzip“ („Aber auch heute ist eine fremde Macht feindselig in mein Inneres gedrungen!“ (Hoffmann 1912b: 116), welches das Wechselspiel von Denken, Fühlen und Handeln bestimmt und vor allem im Traum, „im rein psychischen Wirken des Geistes“(ebd.: 114) das Lauschen der "geheimnisvollen Lauten der Natur" (ebd.: 112) durch die menschliche Seele ausdrucksvoll darstellt. Auch nach Roland Barthes erzeugt der Gesang im Inneren des Menschen ein Reflexionsbild, das die Dynamik seiner Persönlichkeit wiedergibt:

Der Raum des Liedes ist affektiv, er ist kaum sozialisiert: [...] sein wahrer Hörraum ist jedoch, wenn man so sagen kann, das Innere des Kopfes, meines Kopfes: Beim Anhören des Liedes singe ich es mit mir selbst, vor mich selbst hin. Ich wende mich in mir selbst an ein Bild: das Bild des geliebten Wesens, in dem ich mich verliere und aus dem mein eigenes, verlassenes Bild zurückkommt. (Barthes 1990: 289-290)

Den Gesprächspartner des Liedes, die Singstimme, nennt der Philosoph „mein[en] Doppelgänger" - er ist „ein entstellter Doppelgänger, eingefangen in die schreckliche Szene des zersprungenen Spiegels" (Barthes 1990: 290). Wichtig ist daher, was im affektiven Raum des Gesanges mit seinem Empfänger geschieht: Das Nervensystem wird als vibrierende und ertönende „Saiten in unserem Inneren“ beschrieben. Ähnlich spricht J. G. Herder über die menschliche Organisation, die, „wie eine Saite der anderen zutönt“, am meisten dazu gestimmt ist, „den anderen Wesen nachzuhallen und in sich zu fühlen“ (Herder 1989: 345). Immanuel Kant bezeichnet den Gesang in Menschenkunde oder philosophische Anthropologie 
als „eine harmonische Belebung aller Organe“, und „dieser motus tremulus (bebende Bewegung) setzt hernach unser ganzes Nervensystem in eine ähnliche zitternde Bewegung, oder weil er harmonisch zusammenstimmt, den Menschen belebt und gesund erhält" (Kant 1931: 167). Die eigentliche Besitzerin der Singstimme wird bei E. T. A. Hoffmann nicht näher individualisiert, sie bleibt im Bereich des Imaginären verortet, ihre Funktion ist es, dem Erzähler zu seiner inneren Stimme zu verhelfen. Diese Stimme waltet in seinem Inneren und ruft „eine intensive Existenz“ hervor (Hoffmann 1912b: 107). „Heißt doch singen“ - stellt Roland Barthes fest, - „im romantischen Sinn: phantasierend meinen geeinten Leib genieBen“" (Barthes 1990: 288). Es werden „die unbekannten unaussprechlichen Gefühle“ erregt, „welche mit nichts Irdischem hienieden verwandt, die Ahndungen eines fernen Geisterreichs und unsers höhern Seins in demselben hervorrufen" (Hoffmann 1912b: 110), - heißt es in der Erzählung Die Automate. Das Geisterreich oder „unser höheres Sein“ weist auf die tiefsten Tiefen des menschlichen Gemüts, auf das Unbewusste hin, das als ein Resonanzphänomen im Inneren vernehmbar ist und dessen Töne wie „entzückende Strahlen“ hervordringen. Als eine Botin dieses Geisterreiches erscheint die weibliche Singstimme.

Die „ganz wunderherrliche Stimme“ der Sängerin Antonie in der Erzählung Der Rat Krespel wird entsprechend als eine „Phantasie und Gemüt aufregende Sage von einem herrlichen Wunder“" (Hoffmann 1970: 37) gepriesen. Der Erzähler gesteht, dass er „nie eine Ahnung “ hatte, „,on diesen lang ausgehaltenen Tönen, von diesem Steigen bis zur Stärke des Orgellauts, von diesem Sinken bis zum leisesten Hauch“ (ebd.: 36). An anderer Stelle wird der Klang von Antonies Stimme als „Hauch der Äolsharfe, [...] oft dem Schmettern der Nachtigall gleichend“ (ebd.: 48) dargestellt. Ihr Gesang transzendiert das Menschliche, da er ,über die Sphäre des menschlichen Gesanges hinaustönenden Klang gibt“ (ebd.: 48). Die Analogie zum Naturlaut der Nachtigall und zur Äolsharfe macht die Singstimme zur Einheit vermittelnden Universalmusik. Diese Motive verbinden die Sphären von Musik, Psyche, Eros und Tod. Die darin anklingende Entgrenzung bedeutet für Antonie eine spezifische Gefährdung, worauf die Worte hinweisen: „Die Töne schienen nicht Raum haben zu können in der menschlichen Brust" (ebd.: 48). Bedeutsam für die Erzählung ist auch die Analogie zwischen Musikinstrument, Gesangsstimme und Seele. Es handelt sich um eine geheimnisvolle Korrespondenz zwischen Krespels Violine und Antonies Stimme. Als Krespel auf der „Königin“ seiner Geigen spielt, ruft Antonie, „die Königin des Gesangs“, auf: „Ach das bin ich ja - ich singe ja wieder“ (ebd.: 50). Wenn die Töne von Antonies Gesang keinen Raum in der menschlichen Brust zu finden schienen, so klang die Geige, als würde man ihre Töne „in menschlicher Brust erzeugen“. Die durch andere Instrumente vertretene menschliche Stimme ist, laut Roland Barthes, gegenwärtiger, „der Ersatz wird wahrer als das Original" (Barthes 1990: 286). Gerade durch die Verschiebung und Substituierung der Singstimme kommt ihre Bedeutung als ein Sinnesphänomen ausdrucksvoller zur Geltung. Und genau eine solche Substituierung findet in der Erzählung von E. T. A. Hoffmann statt. Der Vater ersetzt die Stimme Antonies durch die Geige (was durch das Gesangsverbot wegen der Krankheit der Tochter erklärt wird) und bringt auf seinem Instrument ihre Stimme ersatzweise zum Erklingen. Erst die Abwesenheit der Stimme Antonies bringt die Rede über ihren Gesang hervor. Der Enthusiast in den Erzählungen von E. T. A. Hoffmann ist ein begeisterter Rezipient des weiblichen Gesanges. Gabriela 
Brandstetter weist darauf hin, dass erst durch die Stimme der Sängerin diese Kunstverständigen zu Schriftstellern werden.

Kunsterlebnis und erotisches Fantasma, Rezeption und Produktion werden so gewissermaßen enharmonisch verwechselt - die Stimme der Sängerin wird zur Schrift des Dichters. Damit aber die Klangbilder durch die Dichtung neu entstehen können, muß die Frau, der Körper der Sängerin, verschwinden. Donna Anna stirbt, damit sie in der Dichtung Don Juan lebt, die Stimme der Sängerin muß verlöschen, um als Stimme der Muse in der Literatur in ihrer Apotheose zu erstrahlen. (Brandstetter 1988: 30-31)

Demnach unterstützt der Gesang in den Erzählungen auch den literarischen Schaffensprozess und erfüllt somit eine Kreativität fördernde Funktion. Ein besonderes Verhältnis zwischen der Gesangsstimme und der Stimme des Erzählers, zwischen Sprache und Musik wird hier noch einmal betont. Nach Antonies Tod baut Krespel keine Geigen mehr, er beginnt zu erzählen, somit wird seine Stimme zur Stimme der Erzählung und Antonies körperliche Eigenschaft der Singstimme zum Text.

Dadurch, dass der Gesang in den Erzählungen von E. T. A. Hoffmann eine besonders intensive Beschäftigung mit der subjektiven Sphäre und mit psychischen Prozessen ermöglicht, kann man ihn als eine bedeutsame Kategorie der romantischen Poetik betrachten und den singenden Menschen der Galerie der wichtigsten romantischen Gestalten hinzufügen. Der romantische Gesang bildet einen Knotenpunkt der bedeutenden Diskurse der Romantik: des musikästhetischen, anthropologischen, des Traum- und des Geschlechter-Diskurses. Das Hoffmann'sche Menschenbild wird durch den Gesang verdeutlicht.

\section{Literatur}

Barthes, Roland (1990): Der romantische Gesang. In: Ders.: Der entgegenkommende und der stumpfe Sinn. Kritische Essays III. Frankfurt a. M.: Suhrkamp, 286-292.

Brandstetter, Gabriele (1988): Die Stimme und das Instrument. Mesmerismus als Poetik in E. T. A. Hoffmanns „Rat Krespel“. In: Dies. (Hg.): Jacques Offenbachs Hoffmanns Erzählungen. Konzeption. Rezeption. Dokumentation. Laaber: Laaber-Verlag, 15-39.

Herder, Johann Gottfried (1989): Ideen zur Philosophie der Geschichte der Menschheit. Frankfurt a. M.: Verlag Deutscher Klassiker.

Hoffmann, Ernst Theodor Amadeus (1908): Beethovens Instrumental-Musik. In: Ders.: Sämtliche Werke. Historisch-kritische Ausgabe mit Einleitungen, Anmerkungen und Lesarten von Carl Georg von Maassen. Bd. 1. Fantasiestücke in Callots Manier. München und Leipzig: Georg Müller, 55-64.

Hoffmann, Ernst Theodor Amadeus (1912a): Der Kampf der Sänger. In: Ders.: Sämtliche Werke. Historisch-kritische Ausgabe mit Einleitungen, Anmerkungen und Lesarten von Carl Georg von Maassen. Bd. 6. Die Serapions-Brüder. Bd. 2. München und Leipzig: Georg Müller, 22-73.

Hoffmann, Ernst Theodor Amadeus (1912b): Die Automate. In: Ders.: Sämtliche Werke. Historischkritische Ausgabe mit Einleitungen, Anmerkungen und Lesarten von Carl Georg von Maassen. Bd. 6. Die Serapions-Brüder. Bd. 2. München und Leipzig: Georg Müller, 87-120. 
Hoffmann, Ernst Theodor Amadeus (1970): Rat Krespel. In: Ders.: Die Serapions-Brüder. Nach dem Text der Erstausgabe unter Hinziehung der Ausgaben von Carl Georg von Maassen und Georg Ellinger. Darmstadt: Wissenschaftliche Buchgesellschaft, 31-57.

Kant, Immanuel (1915): Kritik der praktischen Vernunft. Kritik der Urteilskraft. In: Ders.: Sämtliche Werke. Bd. 2. Leipzig: Felix Meiner.

Kant, Immanuel (1931): Menschenkunde oder philosophische Anthropologie. Leipzig: Bergk.

Käuser, Andreas (1999): Schreiben über Musik. Studien zum anthropologischen und musiktheoretischen Diskurs sowie zur literarischen Gattungstheorie. München: Fink.

Kluge, Carl Alexander Ferdinand (1811): Versuch einer Darstellung des animalischen Magnetismus als Heilmittel. Berlin: C. Salfeld.

Lubkoll, Christine (1995): Mythos Musik. Poetische Entwürfe des Musikalischen in der Literatur um 1800. Freiburg im Breisgau: Rombach.

Lubkoll, Christine / Neumeyer, Harald (Hgg.) (2015): E. T. A. Hoffmann Handbuch. Leben - Werk Wirkung. Stuttgart: Metzler.

Mayer, Paola (2000): Das Unheimliche als Strafe und Warnung. Zu einem Aspekt von E. T. A. Hoffmanns Kritik an der Frühromantik. In: E. T. A. Hoffmann-Jahrbuch, 8. Berlin: Erich Schmidt, 56-68.

Schmidt, Ricarda (2009): Schmerzliches Sehnen und böser Hohn. Ambivalenz in Hoffmanns Darstellung von Künstlern. In: E. T. A. Hoffmann-Jahrbuch (17). Berlin: Erich Schmidt, 20-36.

Schubart, Christian Friedrich Daniel (1969): Ideen zu einer Ästhetik der Tonkunst. Hildesheim: Olms. 